\title{
Serum SCCA-IgM as a predictor of hepatocellular carcinoma in patients with liver cirrhosis
}

\author{
Daniela Buccione $^{1}$, Gianluca Fatti ${ }^{1}$, Andrea Gallotta ${ }^{2}$, Elisabetta Loggi ${ }^{1}$, Roberto Di Donato ${ }^{1}$, \\ Lilia Testa $^{1}$, Carlo Saitta ${ }^{3}$, Valentina Santi ${ }^{1}$, Antonio Di Micoli ${ }^{1}$, Virginia Erroi ${ }^{1}$, Marta Frigerio ${ }^{1}$, \\ Valentina Fazio $^{4}$, Antonino Picciotto ${ }^{4}$, Alessandra Biasiolo ${ }^{5}$, Francoise Degos ${ }^{6}$, Patrizia Pontisso ${ }^{5}$, \\ Giovanni Raimondo ${ }^{3}$, Franco Trevisani ${ }^{*}$
}

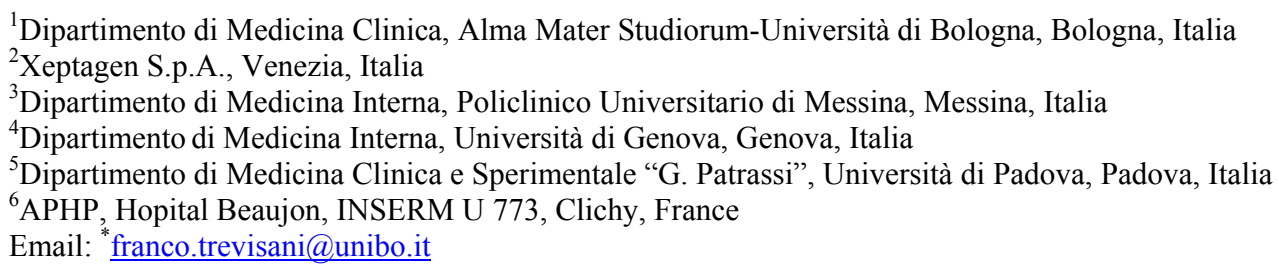

Received 11 January 2012; revised 2 February 2012; accepted 19 February 2012

\begin{abstract}
Aberrant Squamous Cell Carcinoma Antigen (SCCA) expression is an early hepatocarcinogenetic event and circulating SCCA-IgM complexes are elevated in most HCC patients. We evaluated whether serum SCCAIgM levels can identify $\mathrm{HCV}+\mathrm{ve}$ cirrhotic patients at low HCC risk. In this retrospective study we enrolled 29 cirrhotic patients in whom serum SCCA-IgM was measured 8 - 69 months (median 31) before HCC diagnosis, and 28 cirrhotic patients who remained HCCfree, with SCCA-IgM measured 15 - 68 months (median 48 ) before the study end. The best discriminating value of SCCA-IgM was calculated and tested in predicting HCC diagnosis within 12, 24 and 36 months. Sensitivity analysis, considering different HCC incidence, was conducted to identify the patient subgroup with an annual cancer risk below the threshold of a cost-effective semiannual surveillance with ultrasound. Cumulative HCC incidence at 12, 24 and 36 months was $7.0 \%, 15.7 \%$ and $26.3 \%$, respectively. SCCA-IgM levels were higher in $\mathrm{HCC}$ than in cirrhotic patients [median: 381 (95\% C.I.: 50 - 5289) vs. 100 (70 - 493) AU $/ \mathrm{mL}, \mathbf{P}=\mathbf{0 . 0 0 5}]$. The SCCA-IgM value $\leq \mathbf{2 0 0}$ $\mathrm{AU} / \mathrm{mL}$ accurately identified patients at low risk of HCC development in the subsequent year (sensitivity $75 \%$, specificity $62 \%$, positive predictive value $13 \%$ and negative predictive value $97 \%$ ). Considering an annual HCC incidence $\leq 3 \%$, patients with SCCA-IgM $\leq 200 \mathrm{AU} / \mathrm{mL}(60 \%$ of the whole patients) had an HCC risk below the accepted threshold of a cost-effective surveillance $(\mathbf{1 . 5 \%})$. In conclusion, provided that our provocative results are confirmed in larger
\end{abstract}

${ }^{*}$ Corresponding author. studies, SCCA-IgM serum measurement could permit implementation of a two step (with different costs) surveillance: an initial serological surveillance, based on the annual monitoring of this biomarker, and the conventional surveillance by semiannual US when SCCA-IgM becomes $>200 \mathrm{AU} / \mathrm{mL}$. This could improve the cost/effectiveness of surveillance of $\mathrm{HCV}$ infected patients at risk of HCC.

Keywords: SCCA-IgM; HCC Risk Assessment; Surveillance Cost/Effectiveness

\section{INTRODUCTION}

Hepatocellular carcinoma (HCC) is the fifth most common cancer, the third cause of cancer-related death worldwide and the first cause of mortality in cirrhotic patients [1]. Chronic hepatitis $\mathrm{C}$ virus (HCV) infection represents the most important risk factor for $\mathrm{HCC}$ in Western countries and in Japan [2]. In HCV infected cirrhotic patients the annual incidence of HCC ranges from $1 \%$ to $8 \%$, being higher in Japan $(4 \%-8 \%)$, intermediate in Italy $(2 \%-4 \%)$ and lower in the USA $(1.4 \%)$ [3]. The prognosis of HCC patients still remains unsatisfactory, the 5 -year survival rate being less than $10 \%$ both in Europe [4] and the USA [5]. Surveillance of patients at risk of developing $\mathrm{HCC}$, based on the periodic repetition of liver ultrasound (US), makes it possible to detect most cancers at an early stage, still amenable to curative treatments which can greatly improve the prognosis of these individuals [6-10]. Therefore, regular surveillance of patients at risk of $\mathrm{HCC}$ is currently recommended by both Western [11] and Eastern [12,13] practical guide- 
lines for HCC management.

The cost/effectiveness of surveillance is highly dependent on the incidence of HCC and, in the case of cirrhotic patients, decision analysis studies suggest that surveillance becomes cost-effective when the HCC risk is $1.5 \%$ per year or greater $[14,15]$. Nonetheless, a prospective study in Western patients with cirrhosis did not report a satisfactory cost/effectiveness of a semiannual program based on US and alpha-fetoprotein (AFP) determination [7]. This is due to the fact that the risk of HCC development has not yet been clearly defined in an individual basis, and patients with an insufficient risk are included in surveillance. It would therefore be important to exclude these patients in order to save costs. On this prospect, composite systems based on demographic and clinical factors have been proposed to stratify patients according to the HCC risk [16-19] but they have not received external validation and have not entered into clinical practice. To date, no serological marker able to accurately predict tumour development in cirrhotic patients has been found.

Squamous Cell Carcinoma Antigen (SCCA) is a serine protease inhibitor detectable in the spinous and granular layers of normal squamous epithelium but also expressed by neoplastic epithelial cells [20]. An overexpression of SCCA variants (SCCA-1, SCCA-2 and SCCA-PD) has been described in HCC tissue but not in normal liver [21], and a recent study has shown that SCCA is more expressed in cirrhotic tissue adjacent to high grade dysplastic nodules or HCC than in cirrhotic tissue proximal to large regenerative nodules or low grade dysplastic nodules. Thus, SCCA overexpression has been claimed to be an early event in hepatocarcinogenesis [22]. Noteworthy, the serum concentration of circulating immune complexes composed of SCCA and Immunoglobulin M (SCCAIgM IC) paralleled the extent of SCCA overexpression in liver specimens $[23,24]$, and in a small group of $\mathrm{HCV}$ infected patients with cirrhosis progressively increasing levels of serum SCCA-IgM complex were able to identify subjects at higher risk of HCC development [25]. Exploiting such a predictive ability in an opposite way, this biomarker could be utilized to identify inappropriate candidates for surveillance.

Our study aimed at assessing the ability of serum SCCA-IgM levels to segregate, according to the risk of HCC development, HCV infected patients with liver cirrhosis to identify those with a risk below the threshold of a cost-effective US surveillance.

\section{PATIENTS AND METHODS}

\subsection{Patients}

Among cirrhotic patients followed up from April 1994 to January 2010 in 5 Centres, we retrospectively selected those meeting the following inclusion criteria: 1) infected by HCV ( \pm other aetiological factors); 2 ) aged $\geq 50$ years; 3) maintained under semiannual surveillance, based on US ( \pm alpha-fetoprotein determination); 4) availability of one blood sample stored at $-20^{\circ} \mathrm{C}$, collected at least 6 months before HCC occurrence (cases) or the date (January 2009) of study end (controls); 5) control patients were also required to remain tumour-free during the subsequent year; 6) no interferon treatment in the 6 months prior to the collection of the index blood sample.

The 57 patients fulfilling these criteria were divided into 2 groups: HCC group encompassing 29 patients developing HCC during the study period, and control group including 28 patients who remained tumour-free.

\subsection{HCC Diagnosis and Staging}

HCC was diagnosed by histology or according to the non-invasive criteria proposed by the EASL and thereafter the AASLD guidelines [11]. Prior to the availability of these criteria, the non-invasive diagnosis was based on an AFP value $>200 \mathrm{ng} / \mathrm{mL}$ coupled with an imaging work-up suggestive of $\mathrm{HCC}$ and definitely confirmed by the patient follow-up. The tumour was staged as "nonadvanced" if its burden met the Milano criteria or "advanced" if beyond them [26].

\subsection{Serologic Testing}

Circulating SCCA-IgM levels were determined in frozen serum samples, stored at $-20^{\circ} \mathrm{C}$, using an ELISA assay kit (Hepa-IC, Xeptagen SpA, Marghera, Venezia, Italy).

\subsection{Statistical Analysis}

Continuous variables were expressed as mean $\pm \mathrm{SD}$ or median and $95 \%$ C.I. and discrete variables as absolute and relative frequencies.

Continuous non-parametric variables were compared with the Mann-Whitney $\mathrm{U}$ test, and discrete variables with the $\chi^{2}$ test or Fisher's exact test, as appropriate.

The Receiver Operating Characteristic (ROC) curve and the corresponding area under the curve (AUC) were calculated to assess the accuracy of the seromarker in distinguishing HCCs from cirrhosis. The best cut-off value was chosen as the value with the highest Youden index ([sensitivity + specificity] -1 ) and, in the event of equality, the value with higher sensitivity was chosen.

To assess the ability of SCCA-IgM to predict HCC occurrence in clinical practice, we simulated a prospective study protocol with patient assessment every 12 months. The simulation is depicted in Figure 1. In practice, patients who developed HCC during the 12, 24 and 36 months following the SCCA-IgM measurement were shifted to the HCC group, leaving the HCC-free indi- 


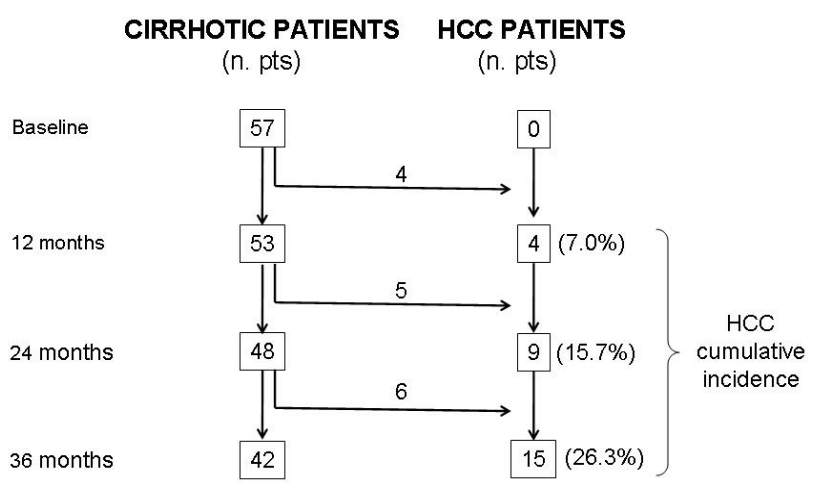

Figure 1. Annual occurrence of hepatocellular carcinoma (HCC) in the studied population at 12, 24 and 36 months from SCCAIgM determination.

viduals in the cirrhotic group. Sensitivity, specificity, positive (PPV) and negative (NPV) predictive values of the SCCA-IgM were calculated. PPV and NPV were also calculated simulating HCC incidences expected in clinical practice.

A sensitivity analysis was then conducted to test the ability of SCCA-IgM to identify, according to HCC incidence in the whole patient population, the subgroup with an HCC risk below 1.5\%/year, which is considered the threshold of a cost-effective surveillance [11].

A 2-tailed $P$ value $<0.05$ was considered statistically significant. Statistical analysis was performed using the SPSS 17.0 statistical package (Chicago, IL), Microsoft Excel program and MedCalc 11 software.

\subsection{Ethics}

The database management conforms to current Italian legislation on privacy and the study conforms to the ethical guidelines of the Declaration of Helsinki. All patients had provided informed consent to register and manage their data in an anonymous way in our database. The study was approved by the ethics committee of the participating Institutions.

\section{RESULTS}

\subsection{Patients' Characteristics (Table 1)}

The HCC group and the control group did not differ for age and gender. In both groups, a few individuals were co-infected with HBV or declared a heavy alcohol intake as cofactors of liver disease. No significant differences were found in the distribution of Child-Pugh classes, with a predominance of patients with well preserved liver function. Most patients did not receive any antiviral therapy, without differences between groups. Nearly $70 \%$ of cases had a non-advanced HCC (within the Milano criteria) at the time of diagnosis.
Table 1. Demographic a clinical characteristics of patients.

\begin{tabular}{lccc}
\hline \multicolumn{1}{c}{ Variable } & $\begin{array}{c}\text { HCC patients } \\
(\mathrm{n}=29)\end{array}$ & $\begin{array}{c}\text { Cirrhotic patients } \\
(\mathrm{n}=28)\end{array}$ & $\mathrm{P}$ \\
\hline Age (years) & $66.5 \pm 8.2$ & $65.8 \pm 10.3$ & n.s. \\
Gender (males) & $18(62.1 \%)$ & $16(57.1 \%)$ & n.s. \\
Aetiology & & & \\
HCV & $25(86.2 \%)$ & $23(82.1 \%)$ & n.s. \\
HCV + HBV & $2(6.9 \%)$ & $3(10.7 \%)$ & \\
HCV + alcohol & $2(6.9 \%)$ & $2(7.1 \%)$ & \\
Child-Pugh class & & & \\
A & $27(93.1 \%)$ & $22(78.6 \%)$ & n.s. \\
B & $2(6.9 \%)$ & $6(21.4 \%)$ & \\
C & $0(0 \%)$ & $0(0 \%)$ & \\
Antiviral therapy & & & \\
no therapy & $28(96.6 \%)$ & $26(92.9 \%)$ & n.s. \\
lamivudine + adefovir & $1(3.4 \%)$ & $2(7.1 \%)$ & \\
Median follow-up (months) & $31(69-8)$ & $48(68-15)$ & n.s. \\
Tumour size (cm) & $2.5 \pm 1.3$ & & \\
Milano criteria & & & \\
within & $20(69.0 \%)$ & & \\
beyond & $9(31.0 \%)$ & & \\
\hline
\end{tabular}

HCV: hepatitis C virus; HBV: hepatitis B virus; SCCA-IgM: Serpin Squamous Cell Carcinoma Antigen-IgM complexes; n.s.: not statistically significant.

\subsection{SCCA-IgM Performance}

Blood samples used for SCCA-IgM determinations were collected from 8 to 69 months (median 31 months) before HCC detection in the HCC group, and from 15 to 68 months (median 48 months) prior to the study end in the control group. Median SCCA-IgM values were higher in the HCC group than in the control group $[381 \mathrm{AU} / \mathrm{ml}$ $(95 \%$ C.I. 117 - 615) vs. $100 \mathrm{AU} / \mathrm{ml}(95 \%$ C.I. 80 - 146); $\mathrm{P}=0.004]$.

The ROC curve of SCCA-IgM levels found from 8 to 69 month prior to the cancer diagnosis (HCC patients) or the end of the study (HCC-free patients) is reported in Figure 2. The best discriminating value of $200 \mathrm{AU} / \mathrm{ml}$ (Youden Index $46.4 \%$ ) had an overall sensitivity of $57.1 \%$, an overall specificity of $89.3 \%$ and, considering the HCC prevalence in our population (51\%), a PPV of $84.2 \%$ and an NPV of $67.6 \%$. The PPV and NPV were also calculated for the cumulative incidence of $\mathrm{HCC}$ observed at 12 (7.0\%), $24(15.7 \%)$ and $36(26.3 \%)$ months after SCCA-IgM measurement (Table 2). Notably, in this model the NPV for HCCs occurring within the year following the SCCA-IgM determination rose to $97 \%$, and it was still $91 \%$ for HCCs occurring within 24 months. When the HCC prevalence was set at 3\% to simulate the annual incidence expected in clinical practice, the NPV for HCC occurrence within 12 and 24 


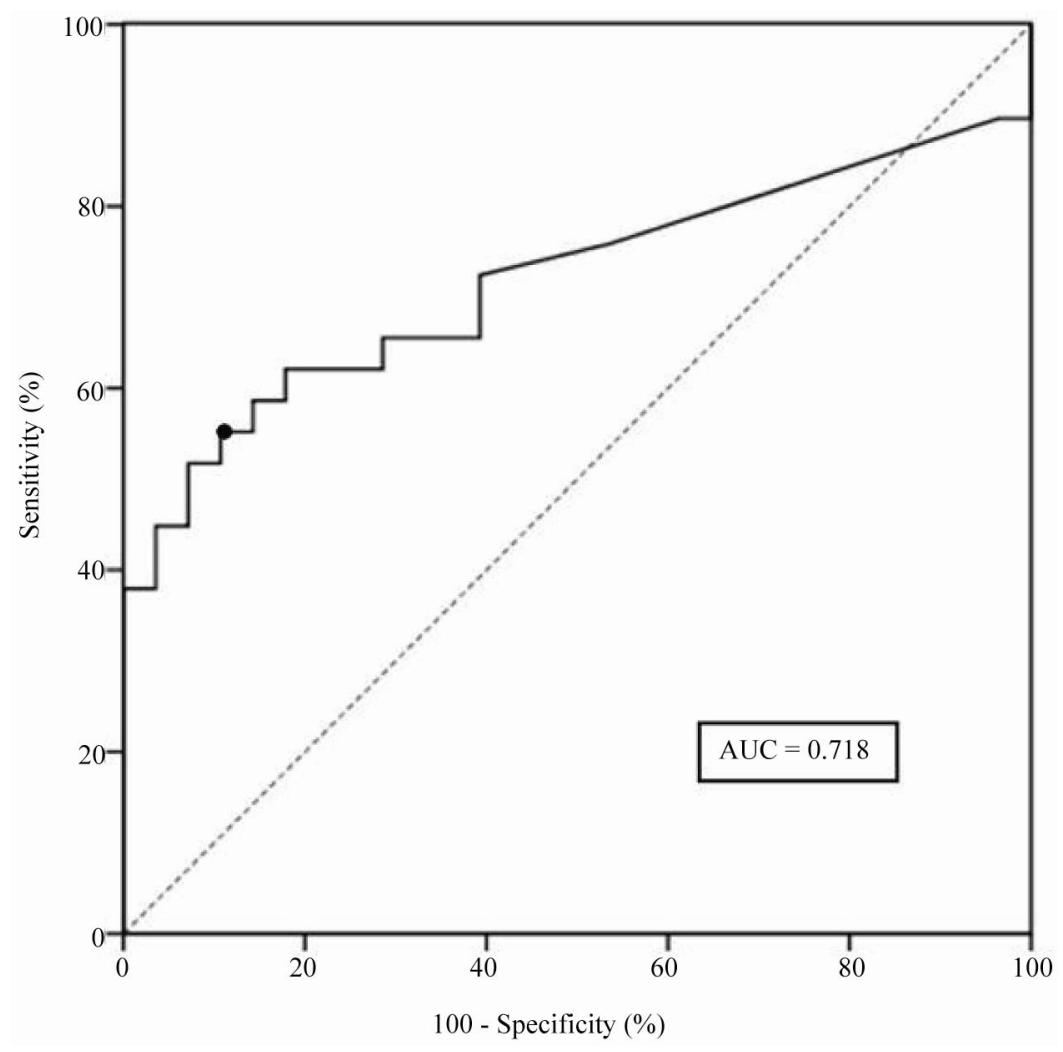

Figure 2. Receiver Operating Characteristic (ROC) curve and corresponding area under the curve (AUC) of SCCA-IgM values measured 8 - 69 months prior to cancer diagnosis (HCC patients) or end of the study (HCC-free patients). The dot indicates the best cut-off value (200 AUA/mL).

Table 2. Sensitivity (Se), Specificity (Sp), Positive (PPV) and Negative (NPV) Predictive Values of the best discriminating value of SCCA-IgM measured 12, 24 and 36 months prior to the HCC occurrence (cases) or the study end (controls).

\begin{tabular}{cccccc}
\hline $\begin{array}{c}\text { Timing } \\
\text { (months) }\end{array}$ & $\begin{array}{c}\text { HCC cumulative } \\
\text { incidence (\%) }\end{array}$ & Se (\%) & Sp (\%) & PPV (\%) & NPV (\%) \\
\hline-12 & 7.0 & 75 & 62 & 13 & 97 \\
-24 & 15.7 & 67 & 65 & 26 & 91 \\
-36 & 26.3 & 60 & 67 & 39 & 82 \\
\hline
\end{tabular}

SCCA-IgM: Serpin Squamous Cell Carcinoma Antigen-IgM complexes.

months further increased to $98.8 \%$ and $98.5 \%$, respectively.

The sensitivity analysis showed that, when annual incidence of $\mathrm{HCC}$ in the whole population was $3 \%$, cirrhotic patients with SCCA-IgM $\leq 200 \mathrm{AU} / \mathrm{ml}$ had a tumour incidence of $1.2 \%$, not enough for implementing a cost-effective semiannual surveillance with US [11] (Table 3). The accepted threshold of $1.5 \%$ was indeed crossed by patients with SCCA-IgM $\leq 200 \mathrm{AU} / \mathrm{ml}$ only when the tumour incidence was at least $4 \%$ in the whole population.
Table 3. Sensitivity analysis according to different annual incidences of HCC. With an overall incidence $\leq 3 \%$, cirrhotic patients with SCCA-IgM $\leq 200 \mathrm{AU} / \mathrm{ml}$ have a risk of developing hepatocellular carcinoma (HCC) below the threshold $(1.5 \%)$ of a cost-effective semiannual surveillance with liver ultrasound.

\begin{tabular}{|c|c|c|}
\hline \multicolumn{3}{|c|}{ Annual incidence of $\mathrm{HCC}(\%)$} \\
\hline $\begin{array}{l}\text { Whole population } \\
\qquad(\mathrm{N}=57)\end{array}$ & $\begin{array}{l}\text { Patients with SCCA-IgM } \\
\leq 200 \mathrm{AU} / \mathrm{ml}(\mathrm{N}=34)\end{array}$ & $\begin{array}{c}\text { Patients with SCCA-IgM } \\
>200 \mathrm{AU} / \mathrm{ml}(\mathrm{N}=23)\end{array}$ \\
\hline 2 & 0.8 & 3.7 \\
\hline 3 & 1.2 & 5.5 \\
\hline 4 & 1.6 & 7.4 \\
\hline
\end{tabular}

\section{DISCUSSION}

Several studies indicate that surveillance of individuals at risk of $\mathrm{HCC}$ increases the chance of detecting tumours at a stage amenable to curative or effective treatments, thus improving the prognosis of these patients [6-9,27]. Since the cost/effectiveness of this procedure is highly dependent on the incidence of $\mathrm{HCC}[14,28]$, the decision to enter a patient into a surveillance program should be determined by his/her level of HCC risk. Nowadays, the individual risk of $\mathrm{HCC}$ development in patients infected 
by HCV (the main cause of this tumour in the Western world and in Japan) has not yet been clearly defined. Therefore, although a Markov model would indicate that semiannual surveillance with US and AFP is cost-effective [28], this assumption has not been confirmed by a prospective observational study, principally including anti-HCV positive patients, where the estimated cost of each year of life gained exceeded $\$ 100,000$ [7]. To optimize the cost-effectiveness of surveillance, we should be able to identify patients in whom surveillance is not worthwhile due to a very low HCC risk. The NPV becomes a crucial indicator for this purpose.

Some predictive indices have been proposed in $\mathrm{HCV}$ infected patients, but these studies do not report the NPV and only considered patients after [29] or during antiviral therapy [30]. The availability of inexpensive, easy to measure serological markers, able to accurately define this risk over a given - and relatively long-time span would be instrumental for this purpose. Since the aberrant expression of SCCA is an early event in hepatocarcinogenesis [22] and the circulating levels of the $\operatorname{IgM}$ immune-complexes parallel the extent of its tissue expression [23], monitoring SCCA-IgM is a promising tool for this perspective.

The prospective approach we simulated made it possible to test the performance of SCCA-IgM measured 12 months prior to $\mathrm{HCC}$ detection. The value of $200 \mathrm{AU} / \mathrm{ml}$ showed a valuable NPV of $97 \%$ one year before cancer diagnosis, despite the high HCC prevalence $(7 \%)$ observed in our model. Moreover, reducing the HCC prevalence to a value simulating the tumour incidence expected in clinical practice (3\%), the NPV reached $98.8 \%$, and the sensitivity analysis revealed that, at this cancer prevalence, the SCCA-IgM can confidently identify HCVinfected cirrhotic patients with a too low risk of developping HCC in the subsequent year to enter a semiannual surveillance based on US. This ability, however, disappears when the HCC incidence in the whole population is $4 \%$ or higher. Therefore, provided that the expected annual risk of $\mathrm{HCC}$ is $\leq 3 \%$ in a given population of $\mathrm{HCV}$ infected patients (as can be anticipated in patients with advanced fibrosis/early cirrhosis), SCCA-IgM serum measurement could permit implementation of two step (with different costs) surveillance: an initial serological surveillance, based on the annual monitoring of this biomarker, and the conventional surveillance by semiannual US when SCCA-IgM becomes $>200 \mathrm{AU} / \mathrm{mL}$ (Figure 3). Using this model, in our series, $34 / 57$ (60\%) cases would have been initially excluded from a non cost-effective semiannual surveillance, and annually postponed until this practice becomes cost-effective, without incurring an unacceptable risk of HCC underdiagnosis (as suggested by an NPV $99 \%$ ).

This pilot study, however, has several limitations: 1) it
HCV-infected patients with an expected annual HCC incidence $\leq 3 \%$

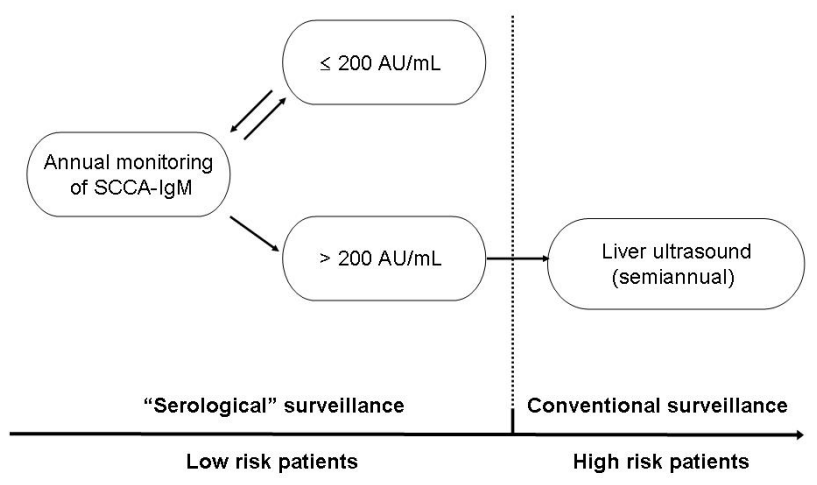

Figure 3. Surveillance algorithm of patients at risk of developing HCC: possible practical application of SCCA-IgM annual monitoring to improve the cost-effectiveness of surveillance of $\mathrm{HCV}$-infected patients with an expected annual $\mathrm{HCC}$ incidence $\leq 3 \%$.

is a retrospective investigation, suffering therefore of the typical methodological biases of these studies; 2) it is based on a small sample size; 3 ) our population included nine patients $(16 \%)$ with HBV infection or heavy alcohol intake as cofactors of liver disease. The small sample size prevented the possibility to obtain reliable results on SCCA-IgM performances in the subgroups. On the other hand, the aetiological panel of our HCV-infected patients reproduces that into which daily clinical practice runs; 4) our results cannot be generalized to patients with non$\mathrm{HCV}$ related cirrhosis or younger than 50 years (excluded from the study).

Therefore, the results of our seminal study, overturning the ordinary use of a new and promising oncomarker from an indicator of a high risk condition to a guide to identify low risk patients, should be considered as a starting point for further prospective investigations aimed at confirming whether SCCA-IgM annual monitoring can improve the cost/effectiveness of surveillance for HCC with an acceptable number of missed early diagnoses.

\section{REFERENCES}

[1] Parkin, D.M., Bray, F., Ferlay, J., et al. (2000) Estimating the world cancer burden: Globocan. International Journal of Cancer, 94, 153-156. doi:10.1002/ijc.1440

[2] Bosch, F.X., Ribes, J., Cléries, R., et al. (2005) Epidemiology of hepatocellular carcinoma. Clinics in Liver Disease, 9, 191-211. doi:10.1016/j.cld.2004.12.009

[3] Fassio, E. (2010) Hepatitis C and hepatocellular carcinoma. Annals of Hepatology, 9, 119-122.

[4] Capocaccia, R., Sant, M., et al. (2007) Hepatocellular carcinoma: Trends of incidence and survival in Europe and the United States at the end of the 20th century. American Journal of Gastroenterology, 102, 1661-1670. 
doi:10.1111/j.1572-0241.2007.01337.x

[5] El-Serag, H.B. (2007) Epidemiology of hepatocellular carcinoma in USA. Hepatology Research, 37, 88-94. doi:10.1111/j.1872-034X.2007.00168.x

[6] Zhang, B.H., Yang, B.H. and Tang, Z.Y. (2004) Randomized controlled trial of screening for hepatocellular carcinoma. Journal of Cancer Research and Clinical Oncology, 130, 417-422. doi:10.1007/s00432-004-0552-0

[7] Bolondi, L., Sofia, S., Siringo, S., et al. (2001) Surveillance programme of cirrhotic patients for early diagnosis and treatment of hepatocellular carcinoma: A cost effecttiveness analysis. Gut, 48, 251-259.

doi:10.1136/gut.48.2.251

[8] Trevisani, F., De Notariis, S., Rapaccini, G., et al. (2002) Semiannual and annual surveillance of cirrhotic patients for hepatocellular carcinoma: Effects on cancer stage and patient survival (Italian experience). American Journal of Gastroenterology, 97, 734-744. doi:10.1111/j.1572-0241.2002.05557.x

[9] Yuen, M.F., Cheng, C.C., Lauder, I.J., et al. (2000) Early detection of hepatocellular carcinoma increases the chance of treatment: Hong Kong experience. Hepatology, 31, 330-335. doi:10.1002/hep.510310211

[10] Stravitz, R.T., Heuman, D.M., Chand, N., et al. (2008) Surveillance for hepatocellular carcinoma in patients with cirrhosis improves outcome. American Journal of Medicine, 121, 119-126. doi:10.1016/j.amjmed.2007.09.020

[11] Bruix, J. and Sherman, M. (2011) Management of heaptocellular carcinoma: An update. Hepatology, 53, 10201022. doi:10.1002/hep.24199

[12] Arii, S., Sata, M., Sakamoto, M., et al. (2010) Management of hepatocellular carcinoma: Report of Consensus Meeting in the 45th Annual Meeting of the Japan Society of Hepatology (2009). Hepatology Research, 40, 667-685. doi:10.1111/j.1872-034X.2010.00673.x

[13] Poon, D., Anderson, B.O., Chen, L.T., et al. (2009) Management of hepatocellular carcinoma in Asia: Consensus statement from the Asian Oncology Summit 2009. Lancet Oncology, 10, 1111-1118. doi:10.1016/S1470-2045(09)70241-4

[14] Sarasin, F.P., Giostra, E., Hadengue, A. (1996) Costeffectiveness of screening for detection of small hepatocellular carcinoma in western patients with Child-Pugh class A cirrhosis. American Journal of Medicine, 101, 422-434. doi:10.1016/S0002-9343(96)00197-0

[15] Arguedas, M.R., Chen, V.K., Eloubeidi, M.A., et al. (2003) Screening for hepatocellular carcinoma in patients with hepatitis $\mathrm{C}$ cirrhosis: A cost-utility analysis. American Journal of Gastroenterology, 98, 679-690. doi:10.1111/j.1572-0241.2003.07327.x

[16] Ganne-Carrié, N., Chastang, C., Chapel, F., et al. (1996) Predictive score for the development of hepatocellular carcinoma and additional value of liver large cell dysplasia in Western patients with cirrhosis. Hepatology, 23, 1112-1118. doi:10.1002/hep.510230527

[17] Degos, F., Christidis, C., Ganne-Carriè, N., et al. (2000) Hepatitis C virus related cirrhosis: Time to occurrence of hepatocellular carcinoma and death. Gut, 47, 131-136. doi:10.1136/gut.47.1.131
[18] Velázquez, R.F., Rodríguez, M., Navascués, C.A., et al. (2003) Prospective analysis of risk factors for hepatocellular carcinoma in patients with liver cirrhosis. Hepatology, 37, 520-527. doi:10.1053/jhep.2003.50093

[19] Yuen, M.F., Tanaka, Y., Fong, D.Y., et al. (2009) Independent risk factors and predictive score for the development of hepatocellular carcinoma in chronic hepatitis B. Journal of Hepatology, 50, 80-88. doi:10.1016/j.jhep.2008.07.023

[20] Kato, H. (1996) Expression and function of squamous cell carcinoma antigen. Anticancer Research, 16, 2149 2153.

[21] Pontisso, P., Calabrese, F., Benvegnù, L., et al. (2004) Overexpression of squamous cell carcinoma antigen variants in hepatocellular carcinoma. British Journal of Cancer, 90, 833-837. doi:10.1038/sj.bjc.6601543

[22] Guido, M., Roskams, T., Pontisso, P., et al. (2008) Squamous cell carcinoma antigen in human liver carcinogenesis. Journal of Clinical Pathology, 61, 445-447. doi:10.1136/jep.2007.051383

[23] Benedice, L., Castaldi, F., Marino, M., et al. (2005) Squamous cell carcinoma antigen-immunoglobulin $\mathrm{M}$ complexes as novel biomarkers for hepatocellular carcinoma. Cancer, 103, 2558-2565. doi:10.1002/cncr.21106

[24] Giannelli, G., Fransvea, E., Trerotoli, P., et al. (2007) Clinical validation of combined serological biomarkers for improved hepatocellular carcinoma diagnosis in 961 patients. Clinica Chimica Acta, 383, 147-152. doi:10.1016/j.cca.2007.05.014

[25] Pontisso, P., Quarta, S., Caberlotto, C., et al. (2006) Progressive increase of SCCA-IgM immune complexes in cirrhotic patients is associated with development of heaptocellular carcinoma. International Journal of Cancer, 119, 735-740. doi:10.1002/ijc. 21908

[26] Mazzaferro, V., Regalia, E., Doci, R., et al. (1996) Liver transplantation for the treatment of small hepatocellular carcinomas in patients with cirrhosis. New England Journal of Medicine, 334, 693-699. doi:10.1056/NEJM199603143341104

[27] Sangiovanni, A., Del Ninno, E., Fasani, P., et al. (2004) Increased survival of cirrhotic patients with a hepatocellular carcinoma detected during surveillance. Gastroenterology, 126, 1005-1014. doi:10.1053/j.gastro.2003.12.049

[28] Lin, O.S., Keeffe, E.B., Sanders, G.D., et al. (2004) Cost-effectiveness of screening for hepatocellular carcinoma in patients with cirrhosis due to chronic hepatitis C. Alimentary Pharmacology and Therapeutics, 19, 11591172. doi:10.1111/j.1365-2036.2004.01963.x

[29] Moriyama, M., Matsumura, H., Aoki, H., et al. (2003) Long-term outcome, with monitoring of platelet counts, in patients with chronic hepatitis $\mathrm{C}$ and liver cirrhosis after interferon therapy. Intervirology, 46, 296-307. doi:10.1159/000073209

[30] Lok, A.S., Seeff, L.B., Morgan, T.R., et al. (2009) Incidence of hepatocellular carcinoma and associated risk factors in hepatitis C-related advanced liver disease. Gastroenterology, 136, 138-148.

doi:10.1053/j.gastro.2008.09.014 